\title{
Dielectric properties of strontium titanate filled mullite composites in microwave region
}

\begin{abstract}
This research was designed to form better dielectric composite material using one steady state dielectric with a good dielectric material. Distinct dielectric composite was successfully produced using locally sourced kaolinite clay. The samples were made using kaolinite as the base matrix and Strontium Titanate (ST) added in varying ratios. Strontium Titanate were synthesized via solid-state reaction using Strontium Carbonate and rutile Titanium (IV) Oxide with sintering at 1300 PC. Local white kaolinite was used to fuse the barium titanate material in varying weight ratios. The powders were dry-mixed and made into pellets for calcination at 1000/PC. The dielectric measurements were carried out using the HP 4291B Impedance Analyzer dielectric setup. Three samples were prepared, namely $10 \% \mathrm{ST}, 20 \% \mathrm{ST}$ and $30 \% \mathrm{ST}$. The dielectric measurements were carried out at room temperature. Microwave region measurements showed steady state and linear dielectric relaxation ranging from 7 in the control sample and dropping down to 5 in $30 \% \mathrm{ST}$. The responses indicate linear relation between $\mathrm{ST}$ addition and microwave region dielectric permittivity.
\end{abstract}

Keyword: CMC; Dielectric measurement; Microwave frequency; Mullite; Strontium titanate 